Commentary

\title{
CeKlotho opens a new road for investigation in worm aging
}

\author{
Florence Solari \\ UM R5201, CNRS, Université Lyon 1, Aging and Tumor Supressor Group, Centre Leon Berard, 69008 Lyon, France
}

Commentary on: Marie-Thérèse Château et al. Klotho interferes with a novel FGF-signalling pathway and insulin/lgf-like signalling to improve longevity and stress resistance in Caenorhabditis elegans. Aging 2010; 2: this issue.

E-mail: solari@lyon.fnclcc.fr

The Klotho (KL) protein was named in reference to the goddess of Greek mythology who spins the thread of life. Mice deficient for KL exhibit many changes that occur during aging, including arteriosclerosis, osteoporosis, infertility, and cognitive decline and have a short life span [1].

The Klotho gene encodes several isoforms in mammals, which include a membrane-bound and two secreted forms. The circulating isoforms result from the proteolytic cleavage of the transmembrane $\mathrm{KL}$ ectodomain (and/or from alternative splicing) and contain KL1 and KL2 sequences, or only KL1, which are homologous to beta-glucosidase.

KL proteins are multifunctional [2]. KL deglycosylates the steroid beta-glucuronides and the calcium chanel transient receptor potential vanilloid 5, TRPV5. It also acts as a cofactor essential for the recognition of fibroblast growth factor FGF 23 which plays a key role in the control of phosphate homeostasis and vitamin D metabolism. The multiple physiological effects of KL also include inhibition of the intracellular insulin/IGF1 receptor (IIRc) signalling cascade. Indeed, mice overexpressing KL live 30\% longer than wild-type mice and show a significant resistance to oxidative stress associated with moderate resistance to insulin/IGF-1, which may partly explain why these mice live longer than wild-type [3]. However the respective role of different $\mathrm{KL}$ isoforms in aging and the molecular mechanisms altered in transgenic mice that are functionally relevant for lifespan regulation are still poorly understood.

In this issue, Château et al. [4] report the first identification and characterization of Klotho genes homologues in Caenorhabditis elegans. Two CeKlotho genes are present in the $C$. elegans genome which both encode predicted proteins with similarity to the beta- glucosidase-like KL1 domain of mammalian KL. RNAi inactivation of CeKlotho reduced wild-type worm's lifespan but did not affect the lifespan of mutants for the IIRc pathway, including mutant for the receptor DAF-2, the PI3K/AGE-1 and the FOXO transcription factor DAF-16. Yamamoto et al. previously published that treatment of mammalian cells with the secreted Klotho protein inhibits IIRc signalling, activates FOXO, induces SOD2 expression and reduces oxidative damages and apoptosis induced by paraquat [5]. In agreement with those data CeKlotho RNAi treated animals were also more sensitive to oxidative stress than control worms. Overall those data support the idea that the regulation of IIRc pathway by KL is conserved between $C$. elegans and mammals and that this pathway constitutes a relevant target for lifespan control by $\mathrm{KL}$ in mice. Interestingly, CeKlotho RNAi did not affect the lifespan of catalytic mutants for IIRc DAF-2 while it reduced the lifespan of mutants in which the ligand binding domain of DAF-2 was mutated. Those results show that CeKL does not require an intact DAF-2 ligand binding domain to inhibit IIRc pathway and thus support the notion that CeKL does not behave as an inhibitory ligand fro DAF-2 (conversely to several characterized ins ligands in C. elegans). Furthermore, genetic data from Château et al. also suggest that CeKL may act both upstream and downstream the IIRc DAF2. Several factors have been shown to impinge on FOXO/DAF-16 in C. elegans. Among others, the betacatenin which functions in the Wnt pathway may deserve more specific attention in light of results obtained in mammals showing that Klotho can also modulate the Wnt signalling.

The membrane-bound isoform of Klotho acts as an obligate co-receptor for FGF23 in the kidney [6]. Interestingly, FGF23 knock out mice exhibits a premature aging syndrome similarly to KL deficient mice. Those data prompted Château et al. to address the 
importance of the FGF Rc pathway for lifespan regulation by CeKL. Conversely to mammals which express multiple FGF ligands and receptors, the $C$. elegans genome encodes only one FGF receptor, EGL15, with two isoforms A and B, and 2 ligands, LET-756 and EGL-17. LET-756 has been involved in fluid balance regulation while EGL-17 has been shown to be required for cell migration of sex myoblasts (SMs). Château et al. showed that similarly to CeKlotho RNAi, mutation of either elg-15 or egl-17 shortened lifespan and increased worm sensitivity to oxidative stress. Furthermore $C e K L$ inactivation in those mutants did not enhance those phenotypes. Those data thus suggest that CeKL and the EGL-17/EGL-15 may function together for lifespan control and oxidative stress resistance. Furthermore they uncover a link between the secreted Klotho isoform and FGF signalling, which so far, was only demonstrated for the membrane-bound isoform in mammals. An attractive hypothesis is that CeKL, via its putative sialidase activity, could de-repress EGL-15 by favouring the removal of inhibitory N-Glycans. Indeed, $\mathrm{N}$-glycosylation has been recently reported to negatively regulate EGL-15 activity in vivo [7].

Overall this study shows that KL function in aging may be at least partly conserved between $C$. elegans and mammals. One of the next steps will be to test whether overexpression of CeKL is sufficient for both increasing worm's lifespan and oxidative stress resistance. This should allow to bypass potential pathological conditions induced by CeKlotho inactivation that may interfere with longevity per se. A major issue highlighted by this work is a potential crosstalk between the IIRc and FGF signalling pathways for lifespan regulation, which clearly deserves further investigations in order to define the functional interaction between the different molecular actors. Thus further study of KL role in $C$. elegans aging should shed light on the secret of goddess Klotho.

\section{REFERENCES}

1. Kuro-o M et al. Nature. 1997; 390:45-51.

2. Wang $Y$ and $Z$ Sun. Ageing Res Rev.2009; 8:43-51.

3. Kurosu H et al. Science. 2005; 309:1829-1833.

4. Château M T et al.Aging. 2010; 2: this issue.

5. Yamamoto $\mathrm{M}$ et al. J Biol Chem. 2005; 280:38029-38034.

6. Urakawa I et al. Nature. 2006; 444:770-774.

7. Polanska, U.M. et al. J Biol Chem. 2009; 284:33030-33039. 